Georgia State University

ScholarWorks @ Georgia State University

\title{
Towards an Experimental Testbed to Study Cyber Worm Behaviors in Large Scale Networks
}

\author{
Harish Kunta \\ Georgia State University \\ Bhavya Induri \\ Georgia State University \\ Anu G. Bourgeois \\ Georgia State University \\ David Maimon \\ Georgia State University \\ Ashwin Ashok \\ Georgia State University
}

Follow this and additional works at: https://scholarworks.gsu.edu/ebcs_presentations

Part of the Criminal Law Commons, Defense and Security Studies Commons, Emergency and Disaster Management Commons, Infrastructure Commons, Internet Law Commons, and the Terrorism Studies

Commons

\section{Recommended Citation}

Kunta, Harish; Induri, Bhavya; Bourgeois, Anu G.; Maimon, David; and Ashok, Ashwin, "Towards an Experimental Testbed to Study Cyber Worm Behaviors in Large Scale Networks" (2020). EBCS Presentations. 2.

https://scholarworks.gsu.edu/ebcs_presentations/2

This Presentation is brought to you for free and open access by the Evidence-Based Cybersecurity Research Group at ScholarWorks @ Georgia State University. It has been accepted for inclusion in EBCS Presentations by an authorized administrator of ScholarWorks @ Georgia State University. For more information, please contact scholarworks@gsu.edu. 
Towards an Experimental

Testbed to Study Cyber

Worm Behaviors in Large

Scale Networks

HARISH KUNTA, BHAVYA INDURI, ANU G. BOURGEOIS,

GeorgaState

DAVID MAIMON, AND ASHWIN ASHOK University. 


\section{Preliminary goals}

- Want a real world experiment to capture true evidence of worm behavior

- Set up experiments in an actual network as opposed to a simulation or testbed environment

- Would capture actual dynamic factors from varying network load

- Identify propagation paths

○ Determine parent-child (infector-victim) relationship

- Determine order of infections 


\section{Preliminary Work}

\begin{tabular}{|c|c|c|}
\hline Node & IP Address & Building \# \\
\hline PI-1 & 10.51 .198 .127 & 26 \\
\hline PI-2 & 10.51 .197 .217 & 26 \\
\hline PI-3 & 10.50 .168 .124 & 37 \\
\hline PI-4 & 10.55 .101 .245 & 27 \\
\hline PI-5 & 10.50 .168 .178 & 37 \\
\hline PI-6 & 10.49 .198 .221 & 25 \\
\hline PI-7 & 10.49 .199 .64 & 25 \\
\hline PI-8 & 10.55 .101 .96 & 27 \\
\hline PI-9 & 10.48 .73 .162 & 6 \\
\hline PI-10 & 10.50 .168 .97 & 37 \\
\hline
\end{tabular}

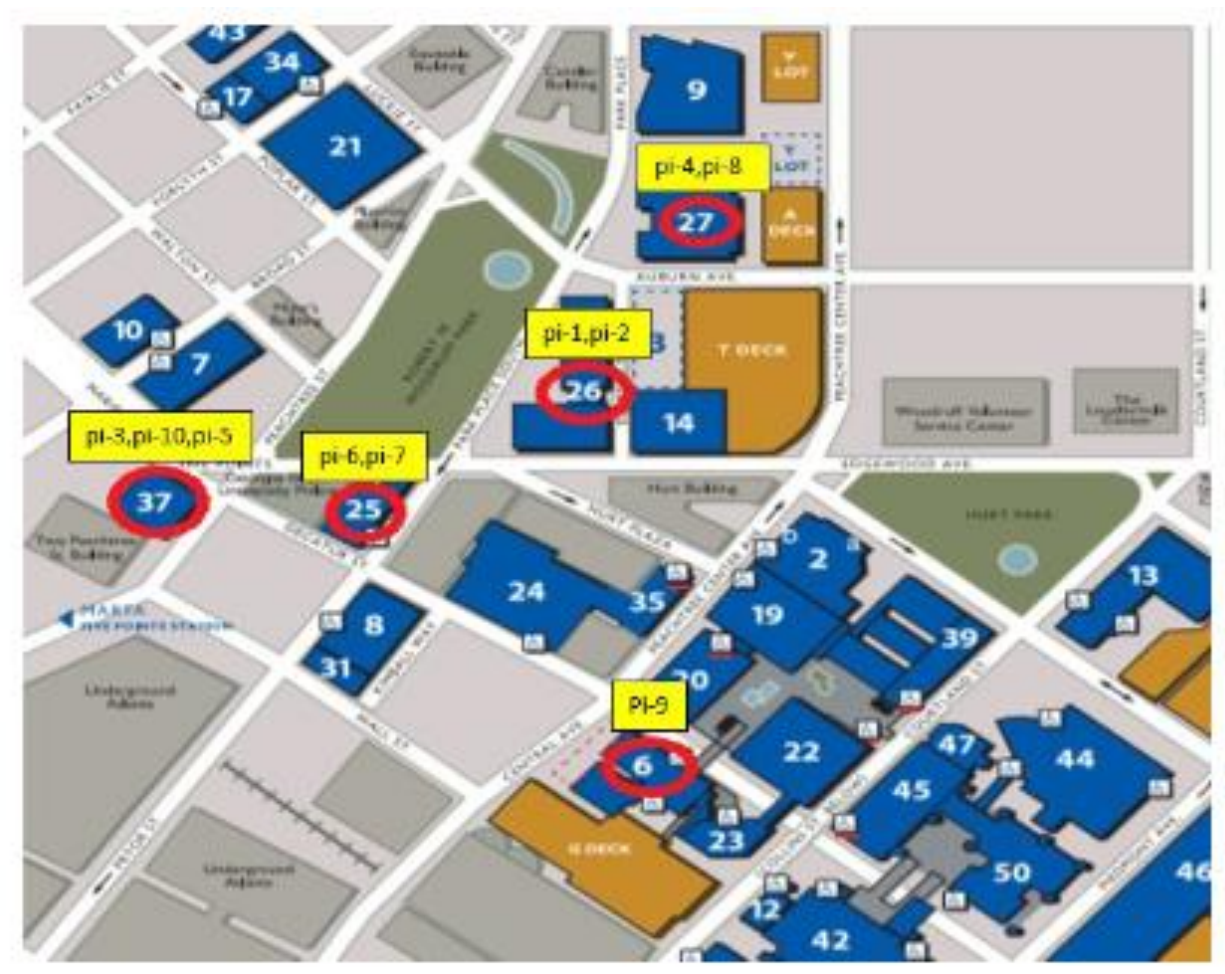




\section{Scanning Cases}

Sequence - Sequence

- Sequentially iterate thru subnets

Sequentially iterate thru hosts

Double nested

Sequence - Random

- Sequentially iterate thru subnets

Randomly select hosts

Double nested
Pseudorandom - Sequence

- Sequentially iterate thru a.b portion

- Randomly select c portion of subnet

- Sequentially iterate thru hosts

- Triple nested

\section{Pseudorandom - Random}

Sequentially iterate thru a.b portion

- Randomly select c portion of subnet

- Randomly select hosts

- Triple nested 


\section{Preliminary results}

\begin{tabular}{|c|c|c|c|c|c|c|c|c|c|}
\hline Node & 2 & 3 & 4 & 5 & 6 & 7 & 8 & 9 & 10 \\
\hline 1 & $(0,0)$ & $(0,0)$ & $(0,0)$ & $(0,0)$ & $(100,0)$ & $(0,0)$ & $(100,0)$ & $(100,100)$ & $(100,0)$ \\
\hline 2 & $(0,0)$ & $(0,0)$ & $(0,0)$ & $(0,0)$ & $(0,100)$ & $(0,0)$ & $(0,0)$ & $(0,0)$ & $(0,0)$ \\
\hline 3 & $(0,0)$ & $(0,0)$ & $(0,0)$ & $(0,0)$ & $(0,0)$ & $(0,100)$ & $(0,0)$ & $(0,0)$ & $(0,0)$ \\
\hline 4 & $(0,0)$ & $(0,0)$ & $(0,0)$ & $(0,0)$ & $(0,0)$ & $(0,0)$ & $(0,0)$ & $(0,0)$ & $(0,100)$ \\
\hline 5 & $(0,0)$ & $(0,100)$ & $(0,0)$ & $(0,24)$ & $(0,0)$ & $(0,0)$ & $(0,0)$ & $(0,0)$ & $(0,0)$ \\
\hline 6 & $(0,0)$ & $(0,0)$ & $(0.7,0)$ & $(100,76)$ & $(0,0)$ & $(0,0)$ & $(0,0)$ & $(0,0)$ & $(0,0)$ \\
\hline 7 & $(100,100)$ & $(0,0)$ & $(0,0)$ & $(0,0)$ & $(0,0)$ & $(0,0)$ & $(0,0)$ & $(0,0)$ & $(0,0)$ \\
\hline 8 & $(0,0)$ & $(0,0)$ & $(0,0)$ & $(0,0)$ & $(0,0)$ & $(0,0)$ & $(0,100)$ & $(0,0)$ & $(0,0)$ \\
\hline 9 & $(0,0)$ & $(100,0)$ & $(99.3,100)$ & $(0,0)$ & $(0,0)$ & $(100,0)$ & $(0,0)$ & $(0,0)$ & $(0,0)$ \\
\hline 10 & $(0,0)$ & $(0,0)$ & $(0,0)$ & $(0,0)$ & $(0,0)$ & $(0,0)$ & $(0,0)$ & $(0,0)$ & $(0,0)$ \\
\hline
\end{tabular}

Sequence - Sequence case

\begin{tabular}{|c|c|c|c|c|c|c|c|c|c|}
\hline Node & 2 & 3 & 4 & 5 & 6 & 7 & 8 & 9 & 10 \\
\hline 1 & $(0.7,0)$ & $(34.5,0)$ & $(47.9,0)$ & $(37.3,0)$ & $(100,0)$ & $(0,0)$ & $(51.4,0)$ & $(100,100)$ & $(28.2,0)$ \\
\hline 2 & $(0,0)$ & $(0,0)$ & $(0,0)$ & $(0,0)$ & $(0,100)$ & $(0,0)$ & $(0,0)$ & $(0,0)$ & $(0,0)$ \\
\hline 3 & $(0,0)$ & $(0,0)$ & $(0.7,0)$ & $(0,0)$ & $(0,0)$ & $(0,100)$ & $(0,0)$ & $(0,0)$ & $(0,0)$ \\
\hline 4 & $(0,0)$ & $(0,34.5)$ & $(0,0)$ & $(0,37.3)$ & $(0,0)$ & $(0,0)$ & $(0,0)$ & $(0,0)$ & $(0,28.2)$ \\
\hline 5 & $(0,0)$ & $(0,47.2)$ & $(0,0)$ & $(0,40.1)$ & $(0,0)$ & $(0,0)$ & $(0,0)$ & $(0,0)$ & $(0,50.7)$ \\
\hline 6 & $(0,0)$ & $(33.8,18.3)$ & $(33.8,0)$ & $(30.3,22.5)$ & $(0,0)$ & $(0,0)$ & $(30.3,0)$ & $(0,0)$ & $(35.9,21.1)$ \\
\hline 7 & $(99.3,100)$ & $(0,0)$ & $(0,0)$ & $(0.7,0)$ & $(0,0)$ & $(0,0)$ & $(0,0)$ & $(0,0)$ & $(0,0)$ \\
\hline 8 & $(0,0)$ & $(0,0)$ & $(0,47.9)$ & $(0,0)$ & $(0,0)$ & $(0,0)$ & $(0,52.1)$ & $(0,0)$ & $(0,0)$ \\
\hline 9 & $(0,0)$ & $(31.7,0)$ & $(17.6,52.1)$ & $(31.7)$ & $(0,0)$ & $(100,0)$ & $(18.3,47.9)$ & $(0,0)$ & $(35.9,0)$ \\
\hline 10 & $(0,0)$ & $(0,0)$ & $(0,0)$ & $(0,0)$ & $(0,0)$ & $(0,0)$ & $(0,0)$ & $(0,0)$ & $(0,0)$ \\
\hline
\end{tabular}

Sequence - Random case

Pseudorandom - Random case

\begin{tabular}{|c|c|c|c|c|c|c|c|c|c|}
\hline Node & 2 & 3 & 4 & 5 & 6 & 7 & 8 & 9 & 10 \\
\hline 1 & $(1.6,0)$ & $(35.3,0)$ & $(51.1,0)$ & $(32.6,0)$ & $(53.3,0)$ & $(46.7,0)$ & $(46.7,0)$ & $(100,100)$ & $(32.1,0)$ \\
\hline 2 & $(0,0)$ & $(0,0)$ & $(0,0)$ & $(0,0)$ & $(0,52.7)$ & $(0,47.3)$ & $(0,0)$ & $(0,0)$ & $(0,0)$ \\
\hline 3 & $(0,0)$ & $(0,0)$ & $(0,0)$ & $(0,0)$ & $(0,47.3)$ & $(0,52.7)$ & $(0,0)$ & $(0,0)$ & $(0,0)$ \\
\hline 4 & $(0,0)$ & $(0,35.9)$ & $(0,0)$ & $(0,32.6)$ & $(0,0)$ & $(0,0)$ & $(0,0)$ & $(0,0)$ & $(0,33.7)$ \\
\hline 5 & $(0,0)$ & $(0,42.9)$ & $(0,0)$ & $(0,45.1)$ & $(0,0)$ & $(0,0)$ & $(0,0)$ & $(0,0)$ & $(0,45.1)$ \\
\hline 6 & $(44,0)$ & $(19,21.2)$ & $(19.6,0)$ & $(21.7,22.3)$ & $(0,0)$ & $(0,0)$ & $(15.2,0)$ & $(0,0)$ & $(15.2,21.2)$ \\
\hline 7 & $(54.4,100)$ & $(12,0)$ & $(1.6,0)$ & $(16.3,0)$ & $(0,0)$ & $(0,0)$ & $(4.9,0)$ & $(0,0)$ & $(17.4,0)$ \\
\hline 8 & $(0,0)$ & $(0,0)$ & $(0,53.3)$ & $(0,0)$ & $(0,0)$ & $(0,0)$ & $(0,48.4)$ & $(0,0)$ & $(0,0)$ \\
\hline 9 & $(0,0)$ & $(33.7,0)$ & $(27.7,46.7)$ & $(29.4,0)$ & $(46.7,0)$ & $(53.3,0)$ & $(33.2,51.6)$ & $(0,0)$ & $(35.3,0)$ \\
\hline 10 & $(0,0)$ & $(0,0)$ & $(0,0)$ & $(0,0)$ & $(0,0)$ & $(0,0)$ & $(0,0)$ & $(0,0)$ & $(0,0)$ \\
\hline
\end{tabular}




\section{Automated Delivery - Robots \& Drones}
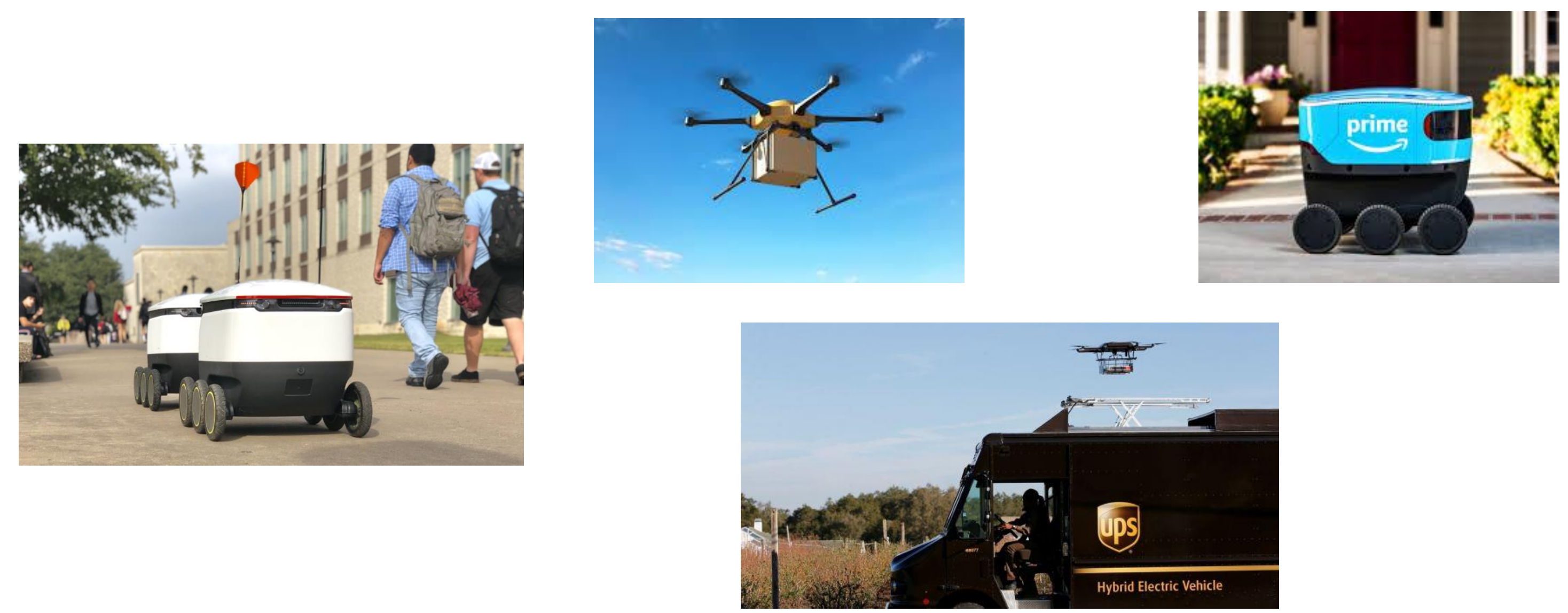


\section{Future goals}

- Set up policies for mitigation of impact or interruption of attack

- Move to a wireless setting for unknown robots/drones coming in as attacker or target

- Maintain privacy and security of data held

- Protect against possible cyberphysical attack of taking over robots 